\title{
Case Consultation: Traumatized Pregnant Woman
}

\author{
Carol Forgash \\ Smithtown, NY \\ Andrew Leeds \\ Santa Rosa, CA \\ Claire A. I. Stramrood \\ University Medical Center, Groningen, The Netherlands \\ Amy Robbins \\ Atlanta, GA
}

\begin{abstract}
Case consultation is a new regular feature in the Journal of EMDR Practice and Research in which a therapist requests assistance regarding a challenging case and responses are written by three experts. In this article, Amy Robbins, a certified eye movement desensitization and reprocessing (EMDR) therapist from Atlanta, Georgia, briefly describes a challenging case in which a pregnant woman seeks treatment for trauma suffered in a tornado. The clinician asks if it is advisable to provide EMDR treatment and what concerns she should be aware of. The first expert, Carol Forgash, provides some general information about pregnancy and psychotherapy and outlines considerations, concerns, and contraindications for proceeding with EMDR. She recommends that if treatment is chosen, the therapist proceed with a recent trauma protocol to specifically target the traumatic memories of the recent tornado. The second expert, Andrew Leeds, comments on the absence of randomized controlled trials (RCTs) or other scientific reports exploring the safety of EMDR treatment of pregnant women. He states that pregnant women with symptoms of posttraumatic stress should understand that there is a high probability that EMDR will improve maternal quality of life and that the risks of adverse effects on stability of pregnancy are probably low, but that these remain unknown. The third expert, Claire Stramrood, explains that the few case studies that evaluated EMDR during pregnancy have found positive effects but pertained to women with posttraumatic stress disorder (PTSD) following childbirth. She asserts that once obstetricians have been consulted, women have been informed about possible risks and benefits, and, given their informed consent, they should be able to choose to commence EMDR therapy during pregnancy.
\end{abstract}

Keywords: EMDR; posttraumatic stress; acute stress disorder; tornado; pregnancy

\section{Therapist's Request}

I have a client "Judy" referred to me by her primary therapist for eye movement desensitization and reprocessing (EMDR). She is 7 months pregnant. She was in a tornado a few months ago in which her entire house was leveled, and the closet in which she and her family were hiding was pulled into the tornado and they "landed" on the street. They were all injured.

Judy has become obsessed, panic filled, frightened... you name it . . . about the weather. She bought hard hats for everyone in the family. When the weather is even slightly rainy and thundering, she escalates into a full-blown panic attack and has everyone hiding, checks the weather several times a day, and becomes hyperfocused when impending "bad weather" is coming.

Judy heard about EMDR and is desperate to do this to ease these issues. I explained to her the liability of doing this with a pregnant woman and she said, "I'm not worried, what I am doing to my baby (extreme stress) is far worse than me doing EMDR." I do have an informed consent. What are your thoughts? Would you do EMDR with her? 


\section{Response From Expert No. 1, Carol Forgash}

I'd like to start by providing some general remarks about pregnancy and psychotherapy. Pregnancy is defined as a normal healthy state for most women: that of carrying an embryo from conception to delivery. Although, until the fourth quarter of the 20th century, it was often pathologized by the medical profession, pregnancy is not an illness.

As a therapist who has treated many pregnant women, I am comfortable considering the use of EMDR with pregnant clients. However, in the EMDR community, I have observed that treating pregnant women is still of concern to many therapists. I base this statement on the large number and frequency of questions about using EMDR with pregnant patients posed to the EMDR Institute LISTSERV.

If therapists are willing to view pregnancy as a normal situation, it is easier to exercise clear clinical judgment. This brings up the issue of countertransference. If the therapist has had a difficult pregnancy and so forth, she may need to seek consultation because her own state of mind may be influential in her decision to use EMDR with pregnant clients. Male therapists who have a spouse or relative who may have had problems while pregnant might also profit from consultation about issues that emerge for them. Providing information about EMDR to the obstetrician or midwife and getting a release from the practitioner can also allay the therapist's concerns. I would also recommend that the therapist have a conversation with the physician and ask them for information about any pertinent issues that might affect the treatment. In addition, the therapist can let the physician know that they understand the implications of posttraumatic stress disorder (PTSD) on the health of the client and explain the need for trauma treatment.

In the case of Judy, where we don't have much information about her prepregnancy and current health, I would say the following: Under normal circumstances, if the client is in good health, the pregnancy is stable, and the therapist has received clearance from the obstetrician to treat the client with EMDR, I would trust and respect her decision to have EMDR treatment. Elevated stress hormones are harmful both to the pregnant woman and her baby (Poggi-Davis $\&$ Sandman, 2006). It appears that Judy has been severely stressed since the tornado and has intrusive symptoms of PTSD. She is correct about needing trauma resolution (Harvey \& Ramirez, 2010) because PTSD can have negative effects on both maternal and fetal health. When conferring with the physician, let them know that you understand implications of PTSD on the health of your client and explain the need for trauma treatment.

I would take a careful history, including any health or past trauma events, including Judy's fertility and pregnancy history over her lifetime, her attitudes toward pregnancy, any symptoms of PTSD, panic disorder, anxiety, and depression. It would also be important to look for any contraindications to treatment. These might include an unstable dissociative disorder or psychosis. Another concern would be a significant lack of partner/spouse/family support. Her relationship with her physician/midwife is also an important element to consider. In terms of medical issues, does she have any conditions which put her at high risk?

Barring these conditions, because there is very little time left in the pregnancy, I would proceed to develop a treatment plan that would include educating her about EMDR, teaching self-soothing and stress-reducing strategies (if necessary), then setting up a target about the tornado experiences and processing them.

If Judy wished to continue in treatment after the delivery because she had a significant prior trauma history, I would explain that other traumatic events could be treated later. The focus of current treatment would be on the tornado and its effects on her.

In addition, because the tornado only occurred a few months ago, I would work with an EMDR recent incident protocol rather than the standard EMDR protocol. This client's continuing experience of distress related to her experiences in the tornado clearly indicates that the effects of the original event have continued into the present day. I would use the Recent Traumatic Episode Protocol (R-TEP; Shapiro \& Laub, 2008). This protocol is understood to integrate unprocessed parts of an entire traumatic episode: the event, experiences that occur after the event, and the possible changing meaning of the original event until the present. R-TEP works with the disturbing images, the event, multiple targets within the event, and the theme of the entire episode. It has the potential to limit associations to only this episode using eye movement desensitization (EMD; Shapiro, 1989), with repeated returns to the target. The goal would be to keep the doors to any earlier traumas closed off.

The R-TEP also stresses preparation and containment strategies such as the self-soothing and stress-reduction exercises mentioned earlier. In the RTEP approach, the client tells the chronological story of the trauma using bilateral stimulation. The next step is to scan the event and report points of disturbance (POD). A target (the image, negative cognition $[\mathrm{NC}]$, positive cognition [PC], validity of cognition [VOC], subjective units of disturbance [SUD], body sensation) 
is developed around each POD. For Judy, some possible points of disturbance might be the scene of the house being leveled, the injuries to her family, or a rainy day. Each would be processed. Finally, the theme of the entire episode would be processed. For this particular pregnant client, this approach would be very appropriate.

\section{Response From Expert No. 2, Andrew M. Leeds}

The question of the safety of EMDR for pregnant women has not been explicitly addressed in the scientific literature. Searches of online databases (PubMed, PsycINFO, and the Francine Shapiro Library) have turned up no published reports that would indicate any danger from EMDR treatment. On the contrary, Stramrood et al. (2012) reported positive clinical outcomes in a case series with three pregnant women who had developed symptoms of posttraumatic stress after traumatic experiences in their first pregnancies. In all three cases, EMDR treatment was provided during a subsequent, second pregnancy in cases referred for treatment by the attending obstetrician.

As an EMDR trainer, over the course of 20 years, I have received a small number of isolated personal reports in which women have complained of unstable pregnancies following EMDR sessions. These women believed that treatment with EMDR was a factor in their subsequent need for bed rest and medical consultation (Leeds, 2009, p. 88). Such reports cannot be considered scientific evidence of risk, but they should not be casually dismissed either. The women who made these reports might have developed unstable pregnancies even if they had not participated in EMDR treatment sessions. Over the course of many consultation groups and workshops, I have observed that the fear of treating pregnant women with EMDR is fairly widespread. This may be caused by potential "risk factors" that were identified in standard EMDR training program manuals from 1991 onward. (Similar unfounded fears seem to apply to offering EMDR to those facing legal procedures or for those with identified genuine epileptic seizures.) Thus, consultation with a woman's obstetrician might not even be encouraged by a clinician who erroneously believed EMDR to be too "dangerous" for a pregnant woman.

EMDR treatment for symptoms of posttraumatic stress is known to be highly effective and efficient (Bisson \& Andrew, 2007). In the absence of scientific study of the safety of EMDR treatment for pregnant women, the probable benefits to maternal quality of life (and reduced stress for the developing fetus) have to be weighed against an unknown degree of safety. Until there are RCTs with a sufficiently large sample of pregnant women, an appropriate discussion would indicate that the risk to stability of pregnancy is probably low but may not be zero, whereas the potential and probable benefits for women with posttraumatic stress symptoms (and their fetuses) are high. Until there are RCTs to provide further guidance on this issue, a conservative approach would suggest that EMDR reprocessing should only be employed with pregnant women after consultation with or upon referral by the woman's physician. At present, it would appear that the same general risks apply to offering EMDR treatment to pregnant women as to all other adults. Appropriate screening for risk factors such as a severe dissociative disorder should take place as always. In Judy's case, the information provided in the summary is insufficient to clearly determine her readiness for EMDR. In particular, no information is provided about her early history, whether there is any history of an anxiety disorder or whether the clinician has screened for dissociative symptoms. In an actual EMDR case consultation, such information would be requested. Once these issues have been clarified and informed consent has been obtained, EMDR should be offered to women who are or who may be pregnant when they are suffering from symptoms for which EMDR is known to be effective.

Judy is eager for EMDR. There are no identified, defined risks to EMDR treatment of Judy or of pregnant women in general. Absent of any specific risk factors not disclosed in the case summary, it would seem a shame to prolong her suffering merely because she is pregnant. Furthermore, at the time of birth, her untreated PTSD symptoms might adversely impact her ability to form a maternal-infant bond (Liotti, 1992; Madrid, Skolek, \& Shapiro, 2006), complicating her subsequent treatment and potentially affecting her newborn child. In my own clinical practice, assuming that she met general readiness criteria, the answer is "yes." I would offer Judy EMDR treatment. In the absence of a referral by her obstetrician (or a history of unstable pregnancy), I would first suggest she discuss her desire for EMDR treatment with her physician, but whether she had that discussion or not, the benefits of EMDR treatment far outweigh the general risks of treatment.

\section{Response From Expert No. 3, Claire A. I. Stramrood}

In many cases of psychological problems during pregnancy, including stress, depression, and anxiety, 
doctors and therapists are faced by a choice between two evils: using treatments that are potentially harmful for the fetus or prolonging the state of being mentally unhealthy with possible adverse effects on the fetus (and pregnant woman) as well.

In my response to this case consultation, I will put forward several arguments in favor of using EMDR in pregnancy. However, in this particular case, I would discuss the risks and benefits for Judy and then leave the decision up to her.

In international and national practice guidelines, trauma-focused cognitive behavioral therapy and EMDR are recommended as the treatments of choice for trauma victims suffering from PTSD or acute stress disorder (American Psychiatric Association, 2004; National Institute for Health and Clinical Excellence [NICE], 2005). The information provided with this case is brief but includes features of both conditions.

A proper diagnosis of Judy's symptoms would be a required first step. Once it has been established that her symptoms are such that she may benefit from EMDR, the question is whether the treatment should be applied during her pregnancy. In the 2001 EMDR book, Shapiro recommends caution because possible hyperarousal and increased stress after the treatment may negatively affect the mother and fetus (p. 96).

Unfortunately, the risks and benefits of applying EMDR during pregnancy have not been well researched. Two pilot studies have been published pertaining to women with PTSD following childbirth. One study used EMDR in three pregnant women (Stramrood et al., 2012), and in another study, four women were studied of which one was pregnant (Sandstrom, Wilberg, Wikman, Willman, \& Hogberg, 2008). Both studies pertained to women suffering from PTSD following childbirth. In other words, the preceding delivery was the traumatic event.

The first study was conducted by the research group that I am part of and took place at a university hospital in the Netherlands. The three women had been traumatized during previous delivery, and they had each developed PTSD related to the birth of their child. They were now pregnant again, showing PTSD symptoms and expressing fear about the upcoming birth. EMDR resulted in stress reduction, fewer posttraumatic stress symptoms, and increased confidence about the upcoming delivery of their second child. Furthermore, despite complications during their second deliveries, all three women had a positive experience of the second birth. With the aforementioned women, we had either direct referral or consultation with the obstetrician, and in my opinion, this is a crucial element.
In this specific case, Judy's trauma and stress are not related to pregnancy or childbirth but to a tornado that the family was in. Considering the fact that Judy is already 7 months pregnant, I can see arguments for postponing the EMDR treatment until after the baby is born. Stress is known to negatively affect fetal neurodevelopment (O’Donnel, O’Connor, \& Glover, 2009), but in all likelihood, most adverse effects of Judy's stress on the fetus have already occurred by 7 months gestation. Another argument would be that EMDR itself may be stress provoking, with possible negative consequences to the pregnancy, as mentioned by Shapiro (2001). However, whether or not treatment has taken place during pregnancy does become relevant in the postpartum period because unresolved trauma/PTSD may negatively affect the mother-baby bond (Parfitt \& Ayers, 2009) and impair secure attachment of the infant (Liotti, 1992).

From Judy's perspective, I can very well imagine that experiencing so much daily stress related to the weather is such a burden that any solution with potentially rapid effects is more than welcome. With that in mind, EMDR would definitely be an option because many people experience its positive effects after only a few sessions.

In conclusion, EMDR during pregnancy has thus far been researched on small scale, with very positive effects for women suffering from PTSD following previous childbirth. In such cases, I would definitely recommend EMDR. Although there is no research on EMDR treatment during pregnancy for women whose trauma is not related to a previous pregnancy and childbirth, the cause of the PTSD is not important, and this is insufficient reason to dissuade or refuse EMDR during pregnancy. Provided that referral by or consultation with the obstetrician has taken place, women have been informed about possible risks and benefits, and, given their informed consent, they should be able to make the choice themselves.

\section{References}

American Psychiatric Association. (2004). Practice guidelines for the treatment of patients with acute stress disorder and posttraumatic stress disorder. Arlington, VA: Author.

Bisson, J., \& Andrew, M. (2007). Psychological treatment of post-traumatic stress disorder (PTSD). Cochrane Database of Systematic Reviews, (3), CD003388.

Harvey, L., \& Ramirez, A. (2010). Hurricane trauma: The effects of prenatal stress on child development. Retrieved from http: / / uwf.edu/argojournal/admin/body/Research Paper-Harvey_\&_Ramirez_Submission_for_Argo_ Journal.pdf 
Leeds, A. M. (2009). A guide to the standard EMDR protocols for clinicians, supervisors, and consultants. New York, NY: Springer Publishing.

Liotti, G. (1992). Disorganized/disoriented attachment in the etiology of the dissociative disorders. Dissociation, 5(4), 196-204.

Madrid, A., Skolek, S., \& Shapiro, F. (2006). Repairing failures in bonding through EMDR. Clinical Case Studies, 5(4), 271-286.

National Institute for Health and Clinical Excellence. (2005). Post-traumatic stress disorder: The management of PTSD in adults and children in primary and secondary care. London, United Kingdom: Author.

O’Donnell, K., O’Connor, T. G., \& Glover, V. (2009). Prenatal stress and neurodevelopment of the child: focus on the HPA axis and role of the placenta. Developmental Neuroscience, 31(4), 285-292.

Parfitt, Y. M., \& Ayers, S. (2009). The effect of post-natal symptoms of post-traumatic stress and depression on the couple's relationship and parent-baby bond. Journal of Reproductive and Infant Psychology, 27(2), 127-142.

Poggi-Davis, E., \& Sandman, C. A. (2006). Prenatal exposure to stress and stress hormones influences child development. Infants and Young Children, 19(3), 246-259.

Sandstrom, M., Wiberg, B., Wikman, M., Willman, A. K., \& Hogberg, U. (2008). A pilot study of eye movement desensitisation and reprocessing treatment (EMDR) for posttraumatic stress after childbirth. Midwifery, 24(1), 62-73.

Shapiro, F. (1989). Eye movement desensitization: A new treatment for post-traumatic stress disorder. Journal of Behavior Therapy and Experimental Psychiatry, 20, 211-217.

Shapiro, F. (2001). Eye movement desensitization and reprocessing. Basic principles, protocols, and procedures (2nd ed.). New York, NY: Guilford Press.

Shapiro, E., \& Laub, B. (2008). Early EMDR intervention (EEI): A summary, a theoretical model, and the recent traumatic episode protocol (R-TEP). Journal of EMDR Practice and Research, 2(2), 79-96.

Stramrood, C. A. I., van der Velde, J., Doornbos, B., Paarlberg, K. M., Weijmar Schultz, W. C. M., \& van Pampus, M. G. (2012). The patient observer: Eye-movement desensitization and reprocessing for the treatment of posttraumatic stress following childbirth. Birth, 39 (1), 70-76.

\section{Meet the Experts}

Carol Forgash is a licensed clinical social worker in private practice in Smithtown, New York specializing in complex trauma. She is an EMDR International Association (EMDRIA)-approved consultant and provider of EMDRIA-approved workshops. She co-edited and contributed to Healing the Heart of Trauma and Dissociation with EMDR and Ego State Therapy, and has written several articles and book chapters about EMDR. She is a past president of the EMDR Humanitarian Assistance Program.

Dr. Andrew M. Leeds, PhD, is a California licensed psychologist and director of training for Sonoma Psychotherapy Training Institute in Santa Rosa, California. He is an EMDRIA- and EMDR Europeapproved consultant and provider of basic training in EMDR. He authored A Guide to the Standard EMDR Protocols for Clinicians, Supervisors, and Consultants (2009) and has written several book chapters and journal articles on EMDR including a research study on resource development and installation (RDI).

Dr. Claire A. I. Stramrood, MD, is a resident in obstetrics / gynecology from Utrecht, the Netherlands. She recently completed a $\mathrm{PhD}$ thesis on women with PTSD following childbirth. She was the main author of a pilot study on EMDR treatment for PTSD following childbirth that was published in 2012, and has been involved in establishing an integrated care model for women with fear of childbirth.

Correspondence regarding this article should be directed to Carol Forgash, LCSW, BCD, 353 North Country Road, Smithtown, NY 11787. E-mail: cforgash@optonline.net 